\title{
TECNOLOGIA CUIDATIVA DE AJUDA MÚTUA GRUPAL PARA PESSOAS COM PARKINSON E SUAS FAMÍLIAS ${ }^{1}$
}

\author{
Edite Lago da Silva Sena ${ }^{2}$,Edméia Campos Meira ${ }^{3}$, Andréa Santos Souza ${ }^{4}$, Isleide Santana Cardoso Santos, ${ }^{5}$ \\ Deusélia Moreira de Souza ${ }^{6}$, Angela Maria Alvarez , Lúcia Hisako Takase Gonçalves ${ }^{8}$
}

${ }^{1}$ Parte do projeto interinstitucional de pesquisa - Tecnologias Assistivas para Autonomia e Inclusão Social de Idoso Portador de Doença de Parkinson - TAIP/FINEP, proc. 23080.031224/2005-39.

2 Doutora em Enfermagem. Professora do Departamento de Saúde da Universidade Estadual do Sudoeste da Bahia (UESB). Bahia, Brasil. E-mail: editelago@gmail.com

${ }^{3}$ Mestre em Enfermagem. Professora do Departamento de Saúde da UESB. Bahia, Brasil. E-mail: edmeiameira@yahoo.com.br

${ }^{4}$ Mestre em Enfermagem. Professora do Departamento de Saúde da UESB. Bahia, Brasil. E-mail: andreasouza_75@yahoo.com.br

${ }^{5}$ Mestranda do Programa de Pós-Graduação de Enfermagem da Universidade Federal da Bahia (UFBA). Professora do Departamento de Saúde da UESB. Bahia, Brasil. E-mail: isleide71@yahoo.com.br

${ }^{6}$ Mestranda do Programa de Pós-Graduação de Enfermagem da UFBA. Professora do Departamento de Saúde da UESB. Bahia, Brasil. E-mail: deuselia@uesb.br

${ }^{7}$ Doutora em Filosofia da Enfermagem. Professora do Departamento de Enfermagem da Universidade Federal de Santa Catarina (UFSC). Santa Catarina, Brasil. E-mail: alvarez@ccs.ufsc.br

${ }^{8}$ Doutora em Enfermagem. Professora do Departamento de Enfermagem da UFSC. Pesquisadora do CNPq. Santa Catarina, Brasil. Coordenadora do projeto TAIP/FINEP. E-mail: lucia@ccs.ufsc.br

\begin{abstract}
RESUMO: Estudo do tipo convergente-assistencial, interinstitucional, que teve o objetivo de adaptar, testar e avaliar a tecnologia assistiva de ajuda mútua grupal para portadores de doença de Parkinson e suas famílias, nos contextos de Jequié-BA e FlorianópolisSC, com foco na inclusão social e formação de rede de relações. Participaram 14 portadores em cada contexto os quais se submeteram ao estudo da efetividade dessa tecnologia e cujo desenvolvimento se deu em dois semestres de 2007. Aplicou-se o Mapa Mínimo de Relações de Sluzki Modificado, que identifica a rede de relações como indicativo de potencial suporte social, no pré e pós-implementação da tecnologia para a aferição das possíveis mudanças como efeito da tecnologia testada. Os resultados apontaram que a tecnologia de ajuda mútua grupal, associada ou não, a outras tecnologias cuidativas, contribui para a manutenção ou alargamento da rede de relações, potencial suporte social favorecedor da inclusão social dos parkinsonianos.
\end{abstract}

DESCRITORES: Tecnologia assistencial. Doença de Parkinson. Grupos de apoio. Idoso.

\section{CARING TECHNOLOGY OF THE MUTUAL HELP GROUP FOR PARKINSON'S DISEASE PATIENTS AND THEIR FAMILIES}

\begin{abstract}
Interinstitutional study of accion research approach, had the objective of adapting, testing and evaluating caretative technology from the mutual help group for patients with Parkinson's disease and their families, in the context of Jequié, BA and Florianopolis, SC by focusing on social inclusion and relations network. Fourteen patients participated in each context, who were submitted to the study of the mentioned technology effectiveness. Its development had place during two semesters in 2007. It was applied the Modified Minimum Map of Relations by Sluzki which identifies the relationships as indicative of potential social support. This application was performed in pre and post technology implementations for data comparison to measure the changes. Results showed that mutual help group technology, associated or not to other caretative technologies, contributes to maintain or enlarge the network of social support and favoring the parkinsonians social inclusion.
\end{abstract}

DESCRIPTORS: Caring technology. Parkinson disease. Self-help groups. Aged.

\section{TECNOLOGÍA CUIDADORA DE AYUDA MUTUA GRUPAL PARA LOS PORTADORES DE ENFERMEDAD DE PARKINSON Y SUS FAMILIAS}

\begin{abstract}
RESUMEN: Estudio interinstitucional convergente de atención tuvo el objetivo de adaptar, testar y evaluar la tecnología cuidativa de ayuda mutua grupal para los portadores de Parkinson y sus respectivas familias, en los contextos de Jequié-BA y Florianópolis-SC con un enfoque en la inclusión social y en la formación de una red de relaciones. Participaron 14 portadores en cada contexto, que se submetieron al estudio de la efectividad de dicha tecnología. Esta fué desarrollada em 2 semestres em 2007. Se aplicó el Mapa Mínimo de Relaciones de Sluzki Modificado que identifica las relaciones como indicativo de potencial soporte social, en pre y post-implementación para comparación de los datos para la evaluación de los cambios. Los resultados mostraron que la tecnología de ayuda mutua grupal, asociada o no a otras tecnologías cuidativas, contribuyó para el mantenimiento o ampliación de la red de potencial soporte social y favorecendo la inclusión social de los parkinsonianos.
\end{abstract}

DESCRIPTORES: Tecnología de cuidado. Enfermidad de Parkinson. Grupos de autoayuda. Anciano.

Texto Contexto Enferm, Florianópolis, 2010 Jan-Mar; 19(1): 93-103. 


\section{INTRODUÇÃO}

Este artigo apresenta resultados de um estudo interinstitucional entre a Universidade Federal de Santa Catarina (UFSC) e Universidade Estadual do Sudoeste da Bahia (UESB) de testagem da tecnologia cuidativa adaptada de desenvolvimento de Grupo de Ajuda Mútua (GAM) para portadores de Doença de Parkinson (DP) e respectivos familiares acompanhantes ou cuidadores. A ação cuidativa grupal tem perspectivas de promover a ajuda mútua no enfrentamento das condições de cronicidade e na inserção ou reinserção social com vistas a manter e/ou alargar a rede de suporte social do binômio portador de DP e sua família.

O gerenciamento de doenças crônicas em meio a condições socioeconômicas desfavoráveis constitui-se em um dos desafios do envelhecimento humano. Por isso, estudos de tecnologias que se traduzam em manutenção da inclusão social das pessoas em condição de cronicidade se tornam essenciais.

A DP é uma afecção crônico-degenerativa e progressiva, de etiologia multifatorial que atinge majoritariamente pessoas idosas, embora seja frequente acometê-las a partir dos 50 anos de idade, beirando atualmente a casa dos 300.000 portadores no Brasil. ${ }^{1}$ A doença atinge o sistema nervoso central, mais especificamente a substância negra, responsável por controlar os movimentos do corpo. A degradação das células neuronais dopaminérgicas acaba por resultar numa gama de sintomatologia, como: tremores, rigidez muscular, bradicinesia, alterações posturais, distúrbios na fala e na deglutição, obstipação, transpiração corporal, incontinência urinaria, depressão. ${ }^{2} \mathrm{O}$ tratamento que pode ser clínico e/ou cirúrgico, visa ao controle dos sintomas por meio de medicamentos, entre os quais se destaca a Levodopa, um precursor da dopamina. Outros grupos de fármacos também são utilizados, como: inibidores enzimáticos, agonistas dopaminérgicos e anti-colinérgicos. Contudo, a terapêutica medicamentosa por si só não é suficiente para manter o portador em melhores condições de bem-estar e qualidade de vida. Por isso, tratamentos e cuidados no mínimo de enfermagem, fisioterapia, fonoaudiologia e educação física são imprescindíveis como recursos complementares para ajudar o portador a preservar sua funcionalidade e permitir-lhe desempenhar as atividades da vida diária e manter o vínculo social, tão importantes para satisfazer suas necessidades psicossociais de apoio comunitário para o seu bem-estar e auto-estima. ${ }^{1}$
Considerando a carência de tecnologias assistivas nos sistemas social e de saúde vigentes, que os torna frágeis e até certo ponto incompetentes no atendimento de demandas para lidar com as questões sociais, políticas e culturais da saúde, principalmente em populações específicas como aqueles em estado de cronicidade progressiva, como portadores de DP, elegemos testar aqui a efetividade de uma tecnologia cuidativa baseada na ajuda mútua entre esses pacientes e suas famílias. Para tal, definimos como objetivos: adaptar a tecnologia cuidativa de ações de ajuda mútua grupal para o contexto de vida dos portadores de DP; testar e avaliar a tecnologia idealizada com foco no suporte social e inclusão social.

A tecnologia cuidativa de ação grupal de ajuda mútua idealizada e aqui desenvolvida, teve fundamento no referencial teórico de compreensão de tecnologia assistiva como sendo toda e qualquer experiência de atendimento profissional sistematizado, inventada para situação de serviços novos, ou reinventada para serviços que requerem adaptação e atualização. O entendimento de tecnologia aqui adotada é a da concepção de tecnologias leve e leve-dura de Merhy, ${ }^{3}$ diferente das convencionais como produção de equipamentos e máquinas. Trata-se de produção de tecnologia de cuidado que se expressa de modo exclusivo o trabalho vivo em ato, como as ações de ajuda mútua grupal com foco na inserção/reinserção social e na ampliação de rede de relações como potencial suporte social dos pacientes participantes do grupo.

Nessa perspectiva, assumiu-se a concepção de GAM como uma estratégia de cuidado grupal, integrando pessoas que compartilham situações de vida similares, como aquelas que sofrem de processos crônico-degenerativos a exemplo de doença de Alzheimer, diabetes melitus, cardiopatias, DP, bem como os respectivos familiares cuidadores. ${ }^{4-5}$ Não obstante seja ainda pouco difundido em nosso meio, o GAM constitui-se programa já adotado em serviços de saúde (ambulatório, unidade básica de saúde, hospital) com significativo impacto no processo de viver de seus integrantes, pois trata-se de espaço apoiado em relações de confiança que possibilita a livre expressão de sentimentos e o compartilhar de vivências, informações sobre a doença e estratégias de cuidado, além da discussão de situações que exigem tomadas de decisão perante as necessidades físicas ou psicossociais. Enfim, promove o apoio para o enfrentamento de perdas progressivas e limitações impostas pelo problema vivenciado, favorecendo a autonomia 
e independência, e o empoderamento das pessoas, resultando na manutenção ou na criação de novos vínculos sociais. Em nossa vivência, o GAM, além de corresponder a um novo espaço de identificação de suporte social para os portadores e seus familiares, favorece ainda a constituição de uma rede ampliada útil às demandas do grupo na perspectiva da integralidade da pessoa. ${ }^{4-7}$

Por suporte social, entendeu-se um apoio de ordem emocional e instrumental baseado em processo interpessoal de proteção caracterizado por troca recíproca em contexto específico, e que resulta em incremento na saúde mental. ${ }^{8}$ Esse recurso de proteção acontece na dinâmica das relações interpessoais de dar e receber, geralmente estabelecido entre pessoas próximas entre si, como familiares, amigos e membros da comunidade circundante. $^{9-11}$ Nesse sentido, o suporte social é benéfico porque previne e favorece o enfrentamento de problemas, reforça a sensação de controle sobre a própria vida, mantém a autonomia e eleva a auto-estima, reduz a ansiedade e estados depressivos, aumenta a sobrevida e a segurança devido às relações afetivas mais íntimas, mantendo a pessoa mais competente para adaptar-se às atribulações da vida. ${ }^{12-13}$ Conhecendo-se as relações da pessoa em determinada circunstância, desde as mais próximas às mais distantes que gravitam em seu entorno, pode-se identificar que cada relação desempenha uma troca de variados benefícios entre si. Essas relações costumam ser espontâneas, porém é possível e até necessário, em muitas ocasiões que sejam estimuladas para a saúde mental das pessoas convivendo na comunidade.

Adaptar e testar a tecnologia cuidativa baseada no GAM traz contribuições na formulação de instrumentais que podem facilitar a reinserção social dos portadores de DP, mesmo daqueles já com limitações funcionais. Outro aspecto relevante é a interdisciplinaridade em gerontologia que o trabalho de ajuda mútua grupal proporciona, de vez que os profissionais de saúde mediadores da proposta se propõem a estudar e compreender a diversidade da experiência humana, e, em parceria com os sujeitos, portadores de DP, encontrar soluções viáveis no atendimento das demandas que emergem do cotidiano. Soma-se ainda a contribuição do aspecto de promoção da cidadania, à medida que no grupo as pessoas são atendidas com respeito, privacidade e sem discriminação por sua condição de cronicidade e de envelhecimento, e têm a garantia de tomar decisões quanto à própria vida, além de sentirem-se mais fortalecidas para reivindicar seus direitos em setores públicos e privados e na sociedade em geral.

\section{METODOLOGIA}

\section{Tipo de estudo}

A testagem e a avaliação da tecnologia cuidativa em questão basearam-se na abordagem convergente-assistencial, caracterizada pela cooperação mútua entre pesquisadores e sujeitos envolvidos em determinada situação, com vistas a introduzir ou renovar práticas de saúde. ${ }^{14}$ Nesse caso específico, houve a necessidade de formar o lócus da prática, ou seja, o GAM com a participação de portadores da DP e seus familiares.

\section{Campo do estudo}

De natureza interinstitucional, o projeto foi replicado em dois contextos: Florianópolis-SC - UFSC/Programa de Pós-Graduação em Enfermagem (PEN) - Grupo de Estudos sobre Cuidados em Saúde de Pessoas Idosas e Jequié-BA - UESB/ Departamento de Saúde (DS) - Grupo de Estudos e Pesquisas do Envelhecimento (GREPE).

Jequié está localizada no sudoeste da Bahia, tem população estimada de 149.258 habitantes, dos quais 15.616 idosos, representa $10,5 \%$ da população total. ${ }^{15}$ As ações específicas de cuidado para portadores de DP praticamente não existiam no município. Esse público começou a integrar-se em grupo e receber atenção a partir deste projeto Tecnologia Assistiva para Autonomia e Inclusão Social do Idoso Portador da Doença de Parkinson (TAIP), quando as pesquisadoras da equipe local empenhararam-se durante um semestre de 2006 a organizar o GAM - sob a forma de projeto de extensão universitária da UESB/DS/GREPE - com a finalidade de servir de campo de estudo para a pesquisa em foco. Assim o GAM reuniu portadores de DP e seus familiares identificando-os de uma lista de usuários que recebiam a medicação específica, disponível na $13^{\text {a }}$ Diretoria Regional de Saúde (DIRES), bem como aqueles diagnosticados, usuários da Clínica de Fisioterapia da UESB. Assim foi formado o lócus da pesquisa com a reunião de potenciais participantes do estudo. ${ }^{16}$

Florianópolis, capital do estado de Santa Catarina, tem população estimada de 416.267 habitantes, dos quais 41.979 idosos, representa 10,1\% da população total. ${ }^{15}$ O GAM de portadores de DP e respectivas famílias desse município é um projeto de extensão universitária que funciona no campus 
da UFSC desde 2005. Trata-se de uma iniciativa de algumas docentes de enfermagem, integrantes do Núcleo da Terceira Idade (NETI) que, atendendo à solicitação da comunidade específica, vêm coordenando as atividades grupais com princípios de mútua ajuda. Seus propósitos são de manter agregados os portadores e acolher nossos membros ao aconchego do grupo, para trocar experiências de vida de enfrentamento da DP, como também de ajudar na organização de empreendimentos, movimentos reinvidicatórios em matérias de interesse do grupo junto ao poder público, como: inclusão de medicamentos específicos atuais na lista de medicamentos especiais do SUS, entre outros. O empoderamento dos parkinsonianos nesse grupo foi tal que, em pouco mais de um ano se organizaram em uma associação denominada Parkinson-SC. ${ }^{17}$ Sua organização e funcionamento, contudo, se faz de modo empírico, exigindo estudo aprofundado quanto a sua efetividade em benefícios aos portadores da doença e sua família.

\section{População alvo e amostra intencional}

A população alvo constituiu-se de portadores de DP que participam do GAM de portadores de DP e suas respectivas famílias de ambos os contextos já citados. Já a amostra, de natureza intencional, foi composta de 14 portadores em cada contexto, integrantes e assíduos participantes do GAM, que se dispuseram a participar assinando o Termo de Consentimento Livre e Esclarecido. Estes submeteram-se às duas avaliações: na pré e na pós-implementação da tecnologia para a aferição de sua efetividade. Portanto o critério de inclusão na amostra obedeceu aos requisitos: participar integralmente da implementação da tecnologia e submeter-se à avaliação pré e pós- implementação da referida tecnologia.

\section{Procedimentos metodológicos}

A implementação do estudo se deu em etapas: idealização da tecnologia adaptando-a a cada contexto com base em alguns exemplos adotados em nosso meio, ${ }^{4-5}$ implementação, avaliação e sucessivas retificações da tecnologia desenhada durante os dois semestres de 2007; avaliação pré e pós-implementação propriamente dita da tecnologia cuidativa, para aferir a efetividade nos parkinsonianos em termos de sua inserção social e manutenção ou expansão da rede de relações em seu entorno.

A realização de cada reunião grupal incluía: abertura para integração e descontração por meio de música, práticas de movimentos corporais motivando os participantes para o momento seguinte, no qual eram socializadas as vivências cotidianas de enfrentamento da doença e das circunstâncias da cronicidade, incentivando as trocas de experiências pessoais e familiares, as quais iriam se configurando como ajuda e apoio para as pessoas. Findava-se a reunião sempre com uma síntese do conteúdo da discussão, coordenados por um ou mais profissionais, que funcionavam como facilitadores do desenvolvimento do GAM. As reuniões quinzenais, oportunizavam seus participantes a comunicarem-se entre si, socializar sentimentos e necessidades e criar laços afetivos e de mútua ajuda. O fortalecimento de vínculos era estimulado tanto por contatos telefônicos entre os pares, quanto para participar de outros grupos de tecnologia cuidativa como vivência corporal, prática de música e reabilitação físico-vocal.

\section{Avaliação da tecnologia cuidativa - instru- mento de coleta de dados}

A implementação do estudo, ou seja, a testagem da tecnologia cuidativa adaptada de ações no GAM, ocorreu após os preparativos realizados em 2006, de instalação e funcionamento efetivo do mesmo, enquanto programa de extensão universitária e lócus da pesquisa. Para avaliar a efetividade da tecnologia cuidativa idealizada a testar, foi adotado o Mapa Mínimo de Relações de Sluzki Modificado (MMRI) ${ }^{18-19}$ que identifica a rede de relações. Essa aplicação deu-se no período pré e pós implementação que se realizou durante os dois semestres de 2007, considerando a necessidade de medidas comparativas para aferir as possíveis mudanças nos parâmetros como efeito da tecnologia testada.

O MMRI (Figura 1) inclui quatro categorias: Família, Amigos, Relações comunitárias e Relações com serviços social e de saúde no qual se podem representar a densidade ou tamanho das relações e o nível de proximidade de cada relação em mais íntima, intermediária e mais distante. A aplicação do instrumento ocorreu perguntando aos portadores de DP: - Quem são as pessoas de relação ou de convivência e de cuidados, e qual o tipo de proximidade? Assinalava-se as respostas no mapa segundo as categorias e as linhas de proximidade. Os dados foram organizados em quadros considerando a distribuição da densidade das relações e os níveis de proximidade das relações obtidos em dois momentos: pré e pós-testagem da tecnologia. 


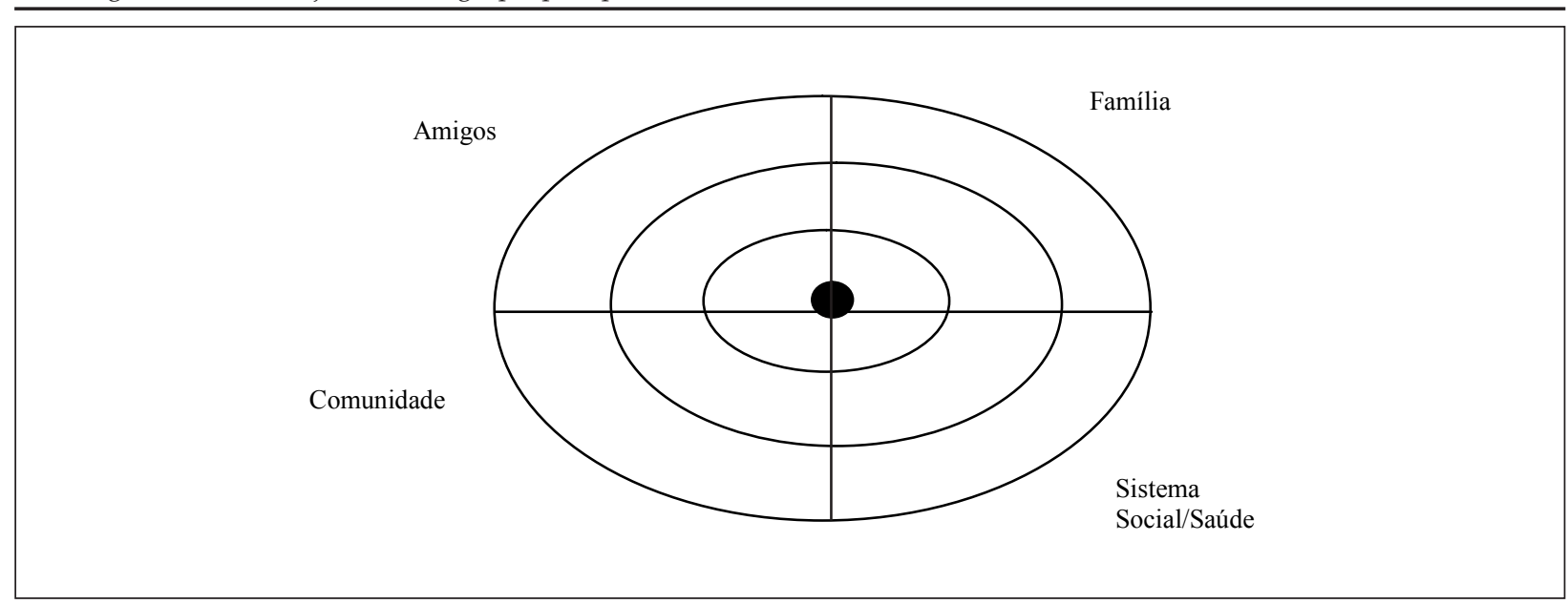

Fonte: Adaptado de Sluski por Domingues (2000)

\section{Figura 1 - Mapa-Mínimo de Relações - MMRI}

Legenda: Família - esposo/a, filha/o, irmã/o, neta/o, prima/o, tia/o, cunhado/a, outros. Amigos - amiga/o. Comunidade - membros do grupo de terceira idade, clubes de serviço e lazer, igreja, Grupo de Ajuda Mútua. Sistema Social/saúde - agente de saúde, profissionais da saúde.

\section{Cuidados éticos segundo Resolução Nº196/96 do CNS/MS}

O projeto foi operacionalizado em consonância com as determinações da Resolução No 196/96 do CNS/MS, que dispõe sobre as normas e diretrizes que regulamentam as pesquisas envolvendo seres humanos. Foi devidamente aprovado pelo Comitê de Ética em Pesquisa com Seres Humanos da UFSC e protocolado sob o $\mathrm{N}^{\mathrm{o}} 350 / 2005$.

\section{RESULTADOS E DISCUSSÃO}

\section{Caracterização da amostra}

Participaram da amostra uma casuística de 14 portadores de DP, em cada contexto. No campus da UESB, eram 9 homens e cinco mulheres; no de UFSC, eram 10 homens e quatro mulheres, confirmando o encontrado na literatura: a DP incide mais em homens do que em mulheres. ${ }^{1-2} \mathrm{~A}$ idade média dos parkinsonianos era de 67 anos, em Jequié a idade variou entre 48 e 77 anos e em Florianópolis, de 55 e 80 anos. Somando-se os dois contextos, foram encontrados seis portadores cujo início da doença se deu na idade entre 48 a 59 anos, confirmando a literatura de incidência de 1 a $2 \%$ em idade precoce, anterior à chegada da velhice. ${ }^{1-2}$ Concernente ao tempo de descoberta da DP, a maioria, referiu conviver com a doença já de seis a até mais de 10 anos. A maioria absoluta dos participantes residia em companhia de seus cônjuges, e quando viúvos/as ou separados/as, geralmente de suas filhas e outros parentes como netos/as e irmãs. Muitos desses familiares eram os que acompanhavam o paciente para frequentar os encontros do GAM e representavam seu real suporte social. Quanto à ocupação, os participantes de Jequié disseram não estar mais na ativa, com exceção de dois comerciantes. Eram: radialista, torneiro mecânico, pedreiro, artesão, relojoeiro e no caso das mulheres, do lar. No contexto de Florianópolis, a maioria aposentados, eram: engenheiro mecânico, engenheiro rodoviário, engenheiro computacional, professor universitário, dentista, funcionário público, pintora, copeira, costureira e, também, do lar. Esses profissionais de nível superior que detêm o poder no exercício do trabalho, mesmo inativos e participantes do GAM, possivelmente se valeram da condição de empoderamento ainda mantida para envolver seus pares e empenharem-se a ponto de constituir a Associação Parkinson - SC, com propósito principalmente, de lutas coletivas de direitos específicos junto aos poderes públicos e sociedade em geral.

\section{Tecnologia cuidativa de ações de ajuda mú- tua e inclusão social}

Considerando o objetivo de avaliar o pressuposto de que a tecnologia cuidativa, o GAM para portadores de DP e seus familiares, estimularia a formação ou a ampliação da rede de suporte social dos parkinsonianos, buscamos verificar essa relação, por meio do MMRI da pré e pós-testagem da tecnologia que identifica a extensão e o nível de proximidade das categorias de pessoas envolvidas na rede de relações. Nas Tabelas 1 e 2 encontram- 
se os dados obtidos quanto à densidade geral da da rede de relações, respectivamente dos contexrede como também das categorias componentes tos de Jequié e Florianópolis.

Tabela 1 - Componentes da rede de relações dos portadores de doença de Parkinson no pré e póstestagem à tecnologia cuidativa. Jequié-BA, 2006/2007

\begin{tabular}{|c|c|c|c|c|c|c|c|c|c|c|}
\hline \multirow{3}{*}{$\begin{array}{l}\text { Sujeito- } \\
\text { portador } \\
\text { de DP }\end{array}$} & \multirow{2}{*}{\multicolumn{2}{|c|}{$\begin{array}{l}\text { Tamanho de } \\
\text { relações }\end{array}$}} & \multicolumn{8}{|c|}{ Categorias de componentes da rede de relações } \\
\hline & & & \multicolumn{2}{|c|}{ Família } & \multicolumn{2}{|c|}{$\begin{array}{l}\text { Profissional } \\
\text { de saúde }\end{array}$} & \multicolumn{2}{|c|}{$\begin{array}{l}\text { Pessoas da } \\
\text { comunidade }\end{array}$} & \multicolumn{2}{|c|}{ Amigos } \\
\hline & Pré & Pós & Pré & Pós & Pré & Pós & Pré & Pós & Pré & Pós \\
\hline 1 & 7 & 7 & 5 & 5 & - & - & 1 & - & 1 & 2 \\
\hline 2 & 24 & 37 & 14 & 11 & 3 & 5 & 2 & 9 & 5 & 12 \\
\hline 3 & 10 & 35 & 5 & 13 & 1 & 5 & 2 & 5 & 2 & 12 \\
\hline 4 & 11 & 10 & 6 & 8 & 2 & - & 1 & - & 2 & 2 \\
\hline 5 & 20 & 34 & 5 & 9 & 6 & 7 & 4 & 10 & 5 & 8 \\
\hline 6 & 11 & 27 & 9 & 10 & - & 1 & 1 & 8 & 1 & 8 \\
\hline 7 & 10 & 7 & 4 & 3 & 2 & 2 & 2 & 1 & 2 & 1 \\
\hline 8 & 18 & 11 & 9 & 4 & 4 & 4 & 3 & 2 & 2 & 1 \\
\hline 9 & 15 & 22 & 7 & 14 & 2 & 2 & 3 & 2 & 3 & 4 \\
\hline 10 & 22 & 20 & 8 & 7 & 2 & 1 & 4 & 5 & 8 & 7 \\
\hline 11 & 8 & 15 & 5 & 10 & 1 & 3 & - & 1 & 2 & 1 \\
\hline 12 & 28 & 16 & 3 & 7 & 3 & 2 & 12 & 1 & 10 & 6 \\
\hline 13 & 22 & 26 & 7 & 11 & 1 & 3 & 11 & 5 & 3 & 7 \\
\hline 14 & 48 & 33 & 25 & 16 & 3 & 7 & 9 & 1 & 11 & 9 \\
\hline
\end{tabular}

Tabela 2 - Componentes da rede de relações dos portadores de doença de Parkinson no pré e póstestagem à tecnologia cuidativa. Florianópolis - SC, 2006/2007

\begin{tabular}{|c|c|c|c|c|c|c|c|c|c|c|}
\hline \multirow{3}{*}{$\begin{array}{l}\text { Sujeito- } \\
\text { portador } \\
\text { de DP }\end{array}$} & \multirow{2}{*}{\multicolumn{2}{|c|}{$\begin{array}{l}\text { Tamanho de } \\
\text { relações }\end{array}$}} & \multicolumn{8}{|c|}{ Categorias de componentes da rede de relações } \\
\hline & & & \multicolumn{2}{|c|}{ Família } & \multicolumn{2}{|c|}{$\begin{array}{l}\text { Profissional } \\
\text { da saúde }\end{array}$} & \multicolumn{2}{|c|}{$\begin{array}{l}\text { Pessoas da } \\
\text { comunidade }\end{array}$} & \multicolumn{2}{|c|}{ Amigos } \\
\hline & Pré & Pós & Pré & Pós & Pré & Pós & Pré & Pós & Pré & Pós \\
\hline 1 & 14 & 16 & 8 & 8 & 2 & 4 & 1 & 1 & 3 & 3 \\
\hline 2 & 10 & 10 & 3 & 3 & 3 & 3 & 2 & 1 & 2 & 3 \\
\hline 3 & 8 & 10 & 2 & 2 & 1 & 2 & 3 & 4 & 2 & 2 \\
\hline 4 & 10 & 7 & 3 & 3 & 2 & 1 & 4 & 2 & 1 & 1 \\
\hline 5 & 13 & 12 & 7 & 7 & 1 & 1 & 3 & 2 & 2 & 2 \\
\hline 6 & 6 & 14 & 4 & 7 & - & 3 & 1 & 1 & - & 3 \\
\hline 7 & 7 & 7 & 4 & 4 & 1 & 1 & 1 & 1 & 1 & 1 \\
\hline 8 & 9 & 9 & 2 & 2 & 4 & 4 & 2 & 2 & 1 & 1 \\
\hline 9 & 11 & 11 & 5 & 5 & 1 & 1 & 4 & 4 & 2 & 2 \\
\hline 10 & 7 & 13 & 2 & 8 & 1 & 1 & 1 & 3 & 3 & 1 \\
\hline 11 & 6 & 6 & 2 & 3 & 1 & - & 2 & 1 & 1 & 2 \\
\hline 12 & 14 & 6 & 6 & 2 & 1 & 1 & 4 & 2 & 3 & 1 \\
\hline 13 & 10 & 11 & 6 & 6 & - & 1 & 2 & 2 & 2 & 2 \\
\hline 14 & 8 & 14 & 3 & 5 & 2 & 2 & 2 & 3 & 1 & 4 \\
\hline
\end{tabular}

Quanto ao número total de pessoas de relação no MMRI em ambos os contextos, verifica-se uma diferença considerável: de sete a 48 pessoas envolvidas com os parkinsonianos em Jequié, para seis a 16 em Florianópolis. Presume-se que essa diferença se deva às características socioculturais 
do povo baiano, mais naturalmente sociável e com um viver baseado em inter-relações pessoais em sua comunidade do que o povo sulino, de Florianópolis, fato observado na prática cotidana pelas equipes desta pesquisa que atuam como enfermeiras nas programações aos portadores de DP e suas famílias, funcionantes nos dois contextos geográficos referidos. Tal diferença traz implicações do aspecto de formação de rede de suporte social a gravitar em torno da pessoa que necessita de apoio e cuidados especiais. Onde há mais pessoas vinculadas por alguma razão, estas podem ser educadas e capacitadas para se tornarem reais fontes de suporte. Já a ausência ou o pequeno número de pessoas envolvidas exige busca ativa de possíveis relações na família e na comunidade, como também uma atenção especial na mobilização de relações mais próximas e mais freqüentes de membros da equipe dos serviços sociais e de saúde, enquanto rede formal de atenção aos usuários.

Na comparação dos dados de avaliação na pré-testagem com aos da pós-testagem, a maioria dos parkinsonianos teve sua rede mantida ou ampliada: oito em Jequié e 10 em Florianópolis. Quanto à distribuição das categorias de pessoas de relação, se constata que a família é a que recebe o maior peso, configurando-se como principal fonte de suporte. Porém, no contexto de Jequié: amigos e membros da comunidade também representam relações numericamente expressivas. Estudos sobre rede de suporte social, tem apontado que, portadores de doenças crônicas tendem, com o passar do tempo, ${ }^{12-13}$ a reduzir seus vínculos afetivos e sociais; contudo, o nosso resultado contraria essa constatação, presumivelmente como efeito positivo das ações empreendidas no GAM, e também de outras tecnologias: vivências corporais e atividades com música e reabilitação, nas quais muitos dos participantes da amostra foram estimulados a frequentar concomitantemente.

Ao longo da implementação do GAM, observou-se que a manutenção ou aumento da rede de relações pode ser atribuído ao interesse e ao envolvimento das pessoas em torno do paciente, devido à oportunidade de adquirir conhecimentos sobre a doença e modalidades terapêuticas; aprendizagem entre pares sobre mecanismos de enfrentamento das limitações impostas pela doença, ou seja, configurando-se como de ajuda mútua para troca de experiências de quem as vive; o gosto por sentir-se útil e ajudar os outros; reconhecimento e valorização das potencialidades do portador de DP; oportunização de espaço de expressão das emoções; facilitação ao acesso aos serviços sociais e de saúde; motivação para as interações sociais e para o exercício da cidadania como ser de direitos; entre outros.

A estrutura de rede de relações sociais pode ser classificada em informais e formais. Estas últimas se constituem geralmente de profissionais da saúde como: médico, enfermeiro, profissional de educação física, assistente social, psicólogo, fisioterapeuta, odontólogo, nutricionista de hospitais, unidades básicas de saúde ou asilos, ou seja, profissionais de saúde em serviços de atendimento público disponibilizados aos usuários. Já as relações informais são constituídas por familiares, amigos, vizinhos, membros de igreja, participantes de grupos de convivência e apoio, entre outros. ${ }^{4,10}$ Embora ambas as relações sejam complementares, num cenário de fragilidade e ineficiência do Estado em lidar com questões sociais e de saúde, destacam-se as relações informais no desenvolvimento de apoio e cuidados de especial importância, a participação da família, como se constatou nos resultados.

Embora não fora o propósito desta pesquisa especificar o tipo de apoio ofertado pela família (instrumental, emocional, de informação e interação positiva), os apoios costumam ser do tipo instrumental ou o cuidado direto. Tal apoio tem sido constatado em diversas pesquisas que envolvem familiares cuidadores de idosos ${ }^{12,20-22}$ com a agravante de, à medida que a situação de fragilidade da pessoa cuidada avança, aumenta a complexidade do processo de cuidar, além de o número de pessoas da família como cuidadoras tende a diminuir, a ponto de limitar-se a uma ou duas pessoas: um cuidador principal e um secundário. ${ }^{20-22}$

Contudo, o presente estudo traz um dado novo que contraria essa constatação, presumivelmente porque os parkinsonianos, participantes do GAM, são acompanhados por seus respectivos familiares cuidadores que nos encontros são estimulados a agregar o maior número possível de pessoas da família em sistema de rodízio nas atividades cuidativas com vistas a reduzir a sobrecarga do cuidador principal e dividir as tarefas de cuidador a favor de uma dinâmica saudável de cuidar em família.

Por ordem de frequência de categorias de pessoas de relação na rede, mesmo em diferente intensidade, aparecem os amigos, os vizinhos, os profissionais do sistema de saúde e pessoas da comunidade, incluindo os companheiros do próprio GAM de outros grupos de convivência. Tal configuração leva a crer que a tecnologia o GAM favorece o estabelecimento de novas rela- 
ções e a manutenção das existentes, validando o seu papel promotor de interação social positiva e educação para um viver mais saudável. Ficou evidente que a proximidade geográfica, no caso dos vizinhos incluídos como amigos, favorece as relações de cooperação e apoios vários, incluindo as atividades instrumentais, corroborando os achados de outras pesquisas. ${ }^{12-13}$

Quanto à relação formal proveniente do Sistema de Saúde, destacou-se o fisioterapeuta como o profissional de maior proximidade, seguido por médico, enfermeiro, profissional de educação física e agentes de saúde entre outros. Os requerimentos terapêuticos de reabilitação e de cuidados específicos de cada parkinsoniano levam-no a buscar compulsoriamente as interações que lhe proporcionem o atendimento em terapêuticas apropriadas. Tais interações passam a ser consideradas e indicadas em proximidade íntima ou intermediaria, pelo próprio paciente como sendo relações sociais significativas e portanto de real suporte social.

Entre as pessoas de relação da comunidade destacadas pelos portadores encontram-se, principalmente, os membros de grupos de convivência para a terceira idade, membros de igrejas e do próprio GAM. Nesse sentido, as ações cuidativas grupais ajudaram a desenvolver a autoconfiança, mobilizando-os a manter ou expandir sua inserção social, participando ativamente e se constituindo cada qual em suporte social recíproco, intercambiando experiências pessoais e trocas de ajuda, como também a construção de vínculos afetivos e de amizade.

No que tange à configuração do mapa mínimo de relações segundo o nível de proximidade das categorias componentes da rede, os dados se encontram nas Tabelas 3 e 4, respectivamente, dos contextos de Jequié e Florianópolis.

Tabela 3 - Nível de proximidade dos componentes da rede de relações dos portadores de doença de Parkinson no pré e pós-testagem à tecnologia cuidativa. Jequié-BA, 2006/2007

\begin{tabular}{|c|c|c|c|c|c|c|c|c|}
\hline \multirow{3}{*}{$\begin{array}{c}\text { Sujeito } \\
\text { portador } \\
\text { de DP }\end{array}$} & \multirow{2}{*}{\multicolumn{2}{|c|}{ Total da rede }} & \multicolumn{6}{|c|}{ Nível de proximidade } \\
\hline & & & \multicolumn{2}{|c|}{ Íntima (próximo) } & \multicolumn{2}{|c|}{ Social (intermediário) } & \multicolumn{2}{|c|}{ Ocasional (distante) } \\
\hline & Pré & Pós & Pré & Pós & Pré & Pós & Pré & Pós \\
\hline 1 & 7 & 7 & 5 & 4 & 2 & 2 & - & 1 \\
\hline 2 & 24 & 37 & 17 & 16 & 5 & 14 & 2 & 7 \\
\hline 3 & 10 & 35 & 4 & 16 & 2 & 12 & 4 & 7 \\
\hline 4 & 11 & 10 & 5 & 5 & 4 & 1 & 2 & 4 \\
\hline 5 & 20 & 34 & 10 & 17 & 4 & 9 & 6 & 8 \\
\hline 6 & 11 & 27 & 8 & 4 & 2 & 11 & 1 & 12 \\
\hline 7 & 10 & 7 & 6 & 5 & 2 & 2 & 2 & - \\
\hline 8 & 18 & 1 & 8 & 3 & 3 & 5 & 7 & 3 \\
\hline 9 & 15 & 22 & 6 & 4 & 4 & 10 & 5 & 8 \\
\hline 10 & 22 & 20 & 16 & 14 & 5 & 3 & 1 & 3 \\
\hline 11 & 8 & 15 & 5 & 6 & 3 & 5 & - & 4 \\
\hline 12 & 28 & 16 & 17 & 8 & 8 & 6 & 3 & 2 \\
\hline 13 & 22 & 26 & 9 & 9 & 10 & 9 & 3 & 8 \\
\hline 14 & 48 & 33 & 8 & 14 & 13 & 9 & 27 & 10 \\
\hline
\end{tabular}

Conforme se observa nos dados de ambos os contextos, por nível de proximidade das relações, guardada a proporção de densidade maior no caso de Jequié, nos três níveis de relações mais íntimas (mais próxima), social (intermediária) e ocasional (distante), a comparação dos dados obtidos na pré e na pós-implementação da tecnologia demonstra estabilidade: a manutenção das relações anteriores reforça o pressuposto do presente estudo de alargamento ou pelo menos de manutenção das relações de possível suporte social já existente. No contexto de Jequié, a densidade da rede total compreendia de sete a 48 pessoas de relação na pré, para sete a 37 pessoas na pós, demonstrando uma insignificante diminuição da rede, considerando a densidade numerosa constatada como possível característica sociocultural própria daquele contexto. Já em Florianópolis, a densidade da rede era menor, de seis a 14 pessoas na pré, para seis a 16 na pós, praticamente uma densidade mantida. 
Tabela 4 - Nível de proximidade dos componentes da rede de relações dos portadores de doença de Parkinson no pré e pós-testagem à tecnologia cuidativa. Florianópolis - SC, 2006/2007

\begin{tabular}{ccccccccc}
\hline \multirow{2}{*}{$\begin{array}{c}\text { Sujeito } \\
\text { portador } \\
\text { de DP }\end{array}$} & Total da rede & \multicolumn{7}{c}{ Nível de proximidade } \\
\cline { 3 - 9 } & & & & \multicolumn{2}{c}{ íntima (próximo) } & Social (intermediário) & Ocasional (distante) \\
\hline 1 & Pré & Pós & Pré & Pós & Pré & Pós & Pré & Pós \\
\hline 2 & 14 & 16 & 5 & 5 & 9 & 11 & - & - \\
3 & 10 & 10 & 7 & 7 & 2 & 2 & 1 & 1 \\
4 & 8 & 10 & 4 & 4 & 2 & 5 & 2 & 1 \\
5 & 10 & 7 & 7 & 2 & 1 & 4 & 2 & 1 \\
6 & 13 & 12 & 3 & 3 & 2 & 2 & 8 & 7 \\
7 & 6 & 14 & 5 & 7 & 1 & 6 & - & 1 \\
8 & 7 & 7 & 4 & 3 & 3 & 3 & 1 & 1 \\
9 & 9 & 9 & 8 & 7 & - & - & 1 & 2 \\
10 & 11 & 11 & 2 & 2 & 5 & 7 & 4 & 2 \\
11 & 7 & 13 & 4 & 2 & 3 & 10 & 0 & 1 \\
12 & 6 & 6 & 5 & 5 & - & 1 & 1 & - \\
13 & 14 & 6 & 8 & 4 & - & 2 & 6 & - \\
14 & 10 & 11 & 1 & 4 & 6 & 7 & 3 & - \\
& 8 & 14 & 5 & 7 & 2 & 3 & 1 & 4 \\
\hline
\end{tabular}

A distribuição das relações nos três níveis de proximidade observada no MMRI de ambos os contextos, é relativamente equitativa em termos numéricos. Contudo, a identificação de representativo suporte social de uma pessoa se faz verificando as relações mais íntimas ou mais próximas. Nesse sentido, verificou-se que tanto em Jequié quanto em Florianópolis, em sua maioria, os parkinsonianos mantiveram ou aumentaram ligeiramente o número de pessoas de relação em nível mais próximo, desde o início até o fim da experiência de participar do GAM, que teve a duração de um ano, em 2007.

A construção de relações íntimas envolve uma história de convivência, trocas afetivas, respeito e mutualidade. As pessoas incluídas no nível íntimo de proximidade distinguem-se das demais por serem fiéis confidentes, capazes de auxiliar na resolução de problemas, demonstrarem conhecimento profundo do outro e reciprocidade, e por isso se constituem em suporte social. ${ }^{4,20-24}$ Contudo, o fato de haver maior número de pessoas da família no nível mais íntimo, principalmente em fase mais avançada da doença, quando o portador se apresenta com maior grau de dependência, pode não resultar de uma decisão deliberada da família em permanecer nesse nível de relação, mas de obrigação no cumprimento de princípios socioculturais e religiosos, recaindo geralmente sobre as mulheres, por identidade com a cultura do cuidado da família e por , imposição de natu- reza religiosa na preservação de valores morais, entre outros.,10,20-22 Nessas circunstâncias, quando não resulte em convívio saudável, a relação não pode se constituir em suporte social para as pessoas necessitadas de cuidados e apoio especiais. Contudo, em espaços favoráveis à formação de rede de suporte social, observa-se que à medida que aumenta a complexidade dos cuidados em função do progresso da doença, as pessoas de relações mais próximas recorrem a outras para auxiliá-las, como membros da comunidade, outros familiares, amigos e profissionais de saúde, o que pode aumentar o número de relações nos níveis intermediário (social) e ocasional (distante), representando relações, mesmo com menor compromisso e sem regularidade, mas ainda assim, um real suporte social, como se constatou, em muitos casos, neste estudo.

\section{CONSIDERAÇÕES FINAIS}

Durante dois semestres a testagem da tecnologia cuidativa baseada na ajuda mútua grupal adaptada para os parkinsonianos e seus familiares acompanhantes/cuidadores, replicada em dois contextos dos campi de UESB/Jequié-BA e UFSC/ Florianópolis-SC, teve sua efetividade avaliada positivamente em termos de formação e manutenção de rede de relação de pessoas que representem real suporte social desses pacientes. Pressupõe-se que tal rede de relações favoreça aos parkinsonianos 
manterem-se inseridos na sociedade mesmo com as limitações impostas pela doença.

Do aspecto da metodologia adotada de pesquisa convergente assistencial, pôde-se constatar ao longo da implementação das ações de ajuda mútua grupal, sinais subjetivos de sua efetividade quanto a facilitar a aceitação da doença, estímulo ao enfrentamento da situação de cronicidade, capacitação para buscas autônomas de recursos e conhecimentos benéficos ao viver diário, estímulo ao desenvolvimento da solidariedade e entreajuda, desenvolvimento de confiança e respeito mútuos, valoração das capacidades presentes e estímulo à auto-confiança, entre outros.

Guardadas as limitações do estudo que abrangeu uma amostra reduzida, seu resultado positivo, ainda que provisório, indica a necessidade de futuros esforços investigativos aprofundados para buscar resultados objetivos que evidenciem as dimensões e a extensão da utilidade e valor da tecnologia cuidativa de ajuda mútua grupal aplicada ao portador de DP e sua família, bem como de estudos de natureza qualitativa para o desvelamento de comportamentos particulares de cuidado para um viver com qualidade e bemestar possível, enfrentando as adversidades da cronicidade imposta pela doença, tanto no seio da família como na comunidade.

\section{REFERÊNCIAS}

1. Reis T. Doença de Parkinson: pacientes, familiares e cuidadores. Porto Alegre (RS): Pallotti; 2004.

2. Pinheiro JES. Distúrbios do movimento: doença de Parkinson e não-Parkinson. In: Freitas EV, Py L, Cançado FAX, Doll J, Gorzoni ML. Tratado de Geriatria e Gerontologia. Rio de Janeiro (RJ): Guanabara-Koogan; 2006.

3. Merhy MM. Saúde: a cartografia do trabalho vivo. São Paulo (SP): Hucitec; 2002.

4. Alvarez AM, Pelzer MT. Sena ES. O valor dos grupos de ajuda mútua para familiares cuidadores de idosos portadores de doença de Alzheimer e outras similares. Rev Ciências da Saúde. 2004 JanDez; 23(1/2):94-8.

5. Alvarez AM, Gonçalves LHT, Schneider P. Grupo de ajuda mútua de familiares de idosos portadores de doença de Alzheimer e Doenças Similares do HU/UFSC. Rev Ciências da Saúde. 2002 Jul-Dez; 21(2):54-6.

6. Barros CA. Grupos de ajuda mútua. In: Zimerman DE, Osório LC. Como Trabalhamos com Grupos. Porto Alegre (RS): Artmed; 2007. p.107-17.

7. Silvermam PR. Mutual helps groups organization and development. Bervely Hills (US): Sage; 1984.
8. Danielson CB. Families helath and illness. In: Danielson CB. Social support and mutual support groups. St Louis (US): Mosby; 1993. p.213-29.

9. Ramos MP. Apoio social e saúde entre idosos. Sociologias. 2002; 7(4):156-75.

10. Rosa TGC. Redes de Apoio Social: In: Litvoc J, Brito FC. Envelhecimento: prevenção e promoção da saúde. São Paulo (SP): Editora Atheneu; 2004.

11. Williams P, Barclay L, Schmied V. Defining social support in context: a necessary step in improving research, intervention and practice. Qual Health Res. 2004 Sep; 14(7):942-60.

12. Sluzki CE. A rede social na prática sistêmica: alternativas terapêuticas. São Paulo (SP): Casa do Psicólogo; 1997.

13. Andrade GRB, Vaitsman J. Apoio social e redes: conectando solidariedade e saúde. Ciênc Saúde Colet. 2002 Out-Dez; 7(4):925-34.

14. Trentini M, Paim L. Pesquisa convergenteassistencial: um desenho que une o fazer e o pensar na prática assistencial em saúde - enfermagem. $2^{\mathrm{a}}$ ed. Florianópolis (SC): Insular; 2004.

15. Instituto Brasileiro de Geografia e Estatística. Dados populacionais de 2000 [online]. Brasilia (DF): IBGE; 2001 [acesso 2010 Fev]. Disponível em: http:/ / www. ibge.gov.br/cidadesat/default.php.

16. Ministério da Saúde (BR). DATASUS. População residente - Santa Catarina, 2006 [online]. Brasília (DF): MS; 2006 [acesso 2008 Dez]. Disponível em: http:/ / tabnet. datasus.gov.br/cgi/tabcgi.exe?ibge/cnv/popsc.def

17. Gonçalves LHT, Alvarez AM, Arruda MC. Pacientes portadores de doença de Parkinson: significado de suas vivências. Acta Paul Enferm. 2007 Jan-Mar; 20(1):62-8.

18. Domingues MARC. Mapa mínimo de relações: adaptação de um instrumento básico para configuração da rede de suporte social do idoso [dissertação]. São Paulo (SP): Universidade de São Paulo. Faculdade de Saúde Pública; 2000.

19. Domingues MARC. Mapa mínimo de relações: validação da adaptação do instrumento básico para configuração da rede de suporte social do idoso [tese]. São Paulo (SP): Universidade de São Paulo. Faculdade de Saúde Pública; 2006.

20. Alvarez AM. Tendo que cuidar: a vivência do idoso e de sua família cuidadora no processo de cuidar e ser cuidado em contexto domiciliar [tese]. Florianópolis (SC): Universidade Federal de Santa Catarina. Programa de Pós-Graduação em Enfermagem; 2001.

21. Santos SMA. Idosos, família e cultura: um estudo sobre a construção do papel do cuidador. Campinas (SP): Alínea; 2003.

22. Sena ELS, Gonçalves LHT. Vivência de familiares cuidadores de pessoas idosas com DA: perspectiva da filosofia de Merleau-Ponty. Texto e Contexto Enferm. 2008 Abr-Jun. 17(2):232-40 
23. Sena ELS, Meira EC, Souza A, Coronago VGM, Gonçalves LHT, Santos E, et al. Tecnologia assistiva de vivências musicais na recuperação vocal de idosos portadores de Doença de Parkinson. Rev Bras Geriatr Gerontol. 2008 Dez; 11(3):341-55.
24. Dias J, Nascimento LC, Mendes, IJM, Rocha, SM. Promoção de Saúde das Famílias de Docentes de Enfermagem: apoio, rede social e papéis na família. Texto e Contexto Enferm. 2007 Out-Dez; 16(4):688-95. 